\title{
Green Synthesis of Silver Nanoparticles by Statistical Experimental Design
}

\author{
Ayşen Aktürk, Melek Erol Taygun, Gültekin Göller, Sadriye Küçükbayrak \\ Istanbul Technical University, Maslak, Istanbul, 34469, Turkey \\ akturkay@itu.edu.tr; erolm@itu.edu.tr; goller@itu.edu.tr; sadriye@itu.edu.tr
}

\section{Extended Abstract}

Chemical synthesis of nanoparticles have environmentally toxic effects or biological hazards since conventional methods for producing nanoparticles involve the use of organic solvents and toxic reducing agents, such as sodium borohydride and $\mathrm{N}, \mathrm{N}$-dimethylformamide [1,2]. To overcome this problem, green synthesis of nanoparticles using natural polymers (i.e. gelatine, dextran, chitosan, heparin and starch) has emerged as a promising solution [1, 3]. Furthermore, it is very difficult to produce stable nanoparticles because of high activity and their small size of metal nanoparticles. Factors such as $\mathrm{pH}$, temperature, electrolytes and solvents cause nanoparticles to aggregate easily. To produce stable nanoparticles, particles may be incorporated into or on the surface of liposomes or biocompatible capping agents (polysaccharides or proteins) [4].

On the other hand, the selection of the heating procedure is another important factor for crystallization of metal nanoparticles [5]. Microwave assisted nanoparticle synthesis is an environmentally friendly method which is simple, fast and economic. Biocompatible capping agents are available at this process [5, 6]. Additionally, the conversion of microwave energy to thermal energy is fast and uniform which results short crystallization time, homogeneous crystallization and sizecontrolled particle production [5-7]. Recently, metal nanoparticles like gold, silver, sulphides and oxides of various elements have been prepared by using microwave heating instead of thermal heating [3].

Within metal nanoparticles, silver nanoparticles are favored in many applications such as catalysis, photonics, microelectronics, photocatalysis, lithography, biosensors, textile, agriculture, surface enhanced raman scattering, optoelectronics and pharmaceuticals [8-10]. The studies carried out with silver nanoparticles have pointed that the non-toxic or toxic nature of silver nanoparticles depends on the selection of capping agent and dosage [2]. For example, while Ag-dendrimer nanocomposites show toxicity, Ag-polysaccharide nanocomposites have non-toxic nature [4]. Soluble starch has been used as both capping agent and reducing agent in microwave assisted green synthesis of silver nanoparticles. Aldehyde groups of soluble starch enable the reduction of silver ions and the reaction kinetics of the reduction of silver ions by using soluble starch can be controlled by changing temperature or the $\mathrm{pH}$ of the solution [11, 12]. In this work, the microwave heating process for the production of the silver nanoparticles was optimized by using response surface methadology based on Box Behnken design. Four significant factors of this process (concentrations of silver salt (AgNO3), capping agent (soluble starch), catalyst (sodium hydroxide $\mathrm{NaOH}$ ) and heating time) were selected as main parameters. The individual and the interactive effects of these parameters on the size of the produced nanoparticles were determined. The characterization studies were performed with Fourier transform infrared spectroscopy (FTIR), Field Emission Scanning Electron Microscopy (FESEM), Dynamic Light Scattering (DLS) and X-ray Diffraction (XRD). The experiments designed with these factors showed that the size of silver nanoparticles change between 20-90 nm having spherical shape. The effects of concentrations of $\mathrm{AgNO} 3$, starch and $\mathrm{NaOH}$ on nanoparticle size were found to be quadratic. It was determined that the interactions between microwave application time with AgNO3 concentration, microwave application time with starch concentration and AgNO3 concentration with $\mathrm{NaOH}$ concentration were effective on nanoparticle size. However, microwave application time did not have a significant impact on nanoparticle size. Results verified the accuracy of the model which provided a simple and effective method for the production of silver nanoparticles with a controllable and predictable nano diameter by using green synthesis method. 


\section{References}

[1] G. Valencia, L. C. O. Vercik, R. Ferrari, and A. Vercik, "Synthesis and characterization of silver nanoparticles using water-soluble starch and its antibacterial activity on Staphylococcus aures," Starch/Stärke, vol. 65, pp. 931-937, 2013.

[2] M. Valodkar, P. S. Rathore, R. N. Jadeja, M. Thounaojam, R. V. Devkar, and S. Thakore, "Cytotoxicity evaluation and antimicrobial studies of starch capped water soluble copper nanoparticles," J.Hazar.Mater., vol. 201-202, pp. 244-249, 2012.

[3] M. Valodkar, S. Modi, A. Pal, and S. Thakore, "Synthesis and antibacterial activity of $\mathrm{Cu}, \mathrm{Ag}$ and $\mathrm{Cu}-\mathrm{Ag}$ alloy nanoparticles: A green approach,” Mater. Res. Bull., vol. 46, no. 3, pp. 384-389, 2011.

[4] M. Valodkar, R. N. Jadeja, M. C. Thounaojam, R. V. Devkar, and S. Thakor, "Biocompatible synthesis of peptide capped copper nanoparticles and biological effect on tumor cells," Mater. Chem. Phy., vol. 128, no. 1-2, pp. 83-89, 2011.

[5] E. Roy, S. Patra, S. Saha, R. Madhuri, and P. K. Sharma, "Shape-specific silver nanoparticles prepared by microwaveassisted green synthesis using pomegranate juice for bacterial inactivation and removal," $R S C A d v$., vol. 5, pp. 9544395442, 2015.

[6] S. Joseph and B. Mathew, "Microwave-assisted facile synthesis of silver nanoparticles in aqueous medium and investigation of their catalytic and antibacterial activities," J.Molec. Liquids, vol. 197, pp. 346-352, 2014.

[7] H. Yin, T. Yamamoto, Y. Wada, and S. Yanagida, "Large-scale and size-controlled synthesis of silver nanoparticles under microwave irradiation," Mater. Chem. Phy., vol. 83, no. 1, pp. 66-70, 2004.

[8] B. Hu, S. Wang, K. Wang, M. Zhang, and S. Yu, "Microwave-Assisted Rapid Facile 'Green' Synthesis of Uniform Silver Nanoparticles: Self-Assembly into Multilayered Films and Their Optical Properties," J. Phy. Chem. C., vol. 112, pp. 11169-11174, 2008.

[9] A. Hebeish, A. El-Shafei, S. Sharafand, and S. Zaghloul, "Novel precursors for green synthesis and application of silver nanoparticles in the realm of cotton finishing," Carbohydrate Poly., vol. 84, no. 1, pp. 605-613, 2011.

[10] S. Navaladian, B. Viswanathan, T. K. Varadarajan, and R. P. Viswanath, "Microwave-assisted rapid synthesis of anisotropic Ag nanoparticles by solid state transformation," Nanotechnology, vol. 19, no. 4:045603, 2008.

[11] P. Raveendran, J. Fu, and S. L. Wallen, "A simple and 'green' method for the synthesis of Au, Ag, and Au-Ag alloy nanoparticles," Green. Chem., vol. 8, pp. 34-38, 2006.

[12] G. A Kahrilas, W. Haggren, R. L. Read, L. M. Wally, S. J. Fredrick, M. Hiskey, A. L. Prieto, and J. E. Owens, "Investigation of Antibacterial Activity by Silver Nanoparticles Prepared by Microwave-Assisted Green Synthesis with Soluble Starch, Dextrose, and Arabinose,” ACS Sust. Chem. Eng., vol. 2, pp. 590-598, 2014. 